Supporting material for:

NlpD links cell wall remodeling and outer membrane invagination during cytokinesis in Escherichia coli

Authors: Mary-Jane Tsang ${ }^{1}$, Anastasiya A. Yakhnina ${ }^{1}$ and Thomas G. Bernhardt ${ }^{1 *}$

\author{
Affiliations: \\ ${ }^{1}$ Department of Microbiology and Immunobiology \\ Harvard Medical School \\ Boston, MA 02115 \\ *To whom correspondence should be addressed. \\ Thomas G. Bernhardt, Ph.D. \\ Harvard Medical School \\ Department of Microbiology and Immunobiology \\ Boston, Massachusetts 02115 \\ e-mail: thomas bernhardt@hms.harvard.edu
}




\section{Plasmid Construction}

Plasmids used in this study are listed below. PCR was performed using KOD polymerase (Novagen) for cloning purposes and Taq DNA polymerase (NEB) for diagnostic purposes, both according to the manufacturer's instructions. Unless otherwise indicated, MG1655 chromosomal DNA was used as the template. Restriction sites for use in plasmid constructions are bold, italicized and underlined in the primer sequences given below. Plasmid DNA and PCR fragments were purified using the Zyppy plasmid miniprep kit (Zymo Research) or the Qiaquick PCR purification kit (Qiagen), respectively.

pMT12

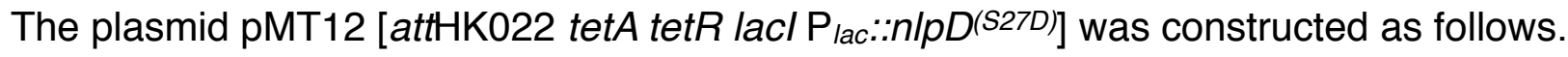
The primers 5'-GACTCTCTTCCGGGCGCTATC-3'/5'GTGCCGGTGGATTTGAAGTGTCATCACAGCCTGC-3' and 5'GTTTCGCTATGGCTGGCAGGCTGTGATGACACTTC-3'/5'GACGAAAGTGATTGCGCCTACC-3' were used in overlap extension PCR to amplify the $n / p D$ gene from pTD23 and to perform site directed mutagenesis to create the S27D mutation in the $n l p D$ gene. The resulting PCR product was purified, digested with Xbal and HindIII and ligated with similarly digested pNP20 [attHK022 tetA tetR lac/a Plac:::nlpD-mCherry] [5].

pMT18 
The plasmid pMT18 [attHK022 bla laclq $\mathrm{P}_{\text {lac:.: }}{ }^{s s} d s b A-n / p D^{(27-379)}$ ] was constructed as follows. The primers 5'-GTCAGGATCCTCTGACACTTCAAATCCACCGGC-3' and 5'GTCA_AAGCTTCCGCCGATTTATCGCTGC-3' were used to amplify nIpD(27-379) from genomic DNA. The resulting PCR product was purified, digested with Xbal and Xhol and ligated with similarly digested pTB282 [attHK022 bla lacla $\mathrm{P}_{\text {lac:: }}$ ssdsbA-sfGFP] [5].

pMT21

The plasmid pMT21 [attHK022 tetA tetR lacl Plac::nlpD (S27D)-mCherry] was constructed as follows. The primers 5'-TTAGGCACCCCAGGCTTTACAC-3' and 5'-

GTCACTCGAGTCGCTGCGGCAAATAACGC-3' were used to amplify $n / p D(S 27 D)$ from pMT12. The resulting PCR product was purified, digested with Xbal and Xhol and

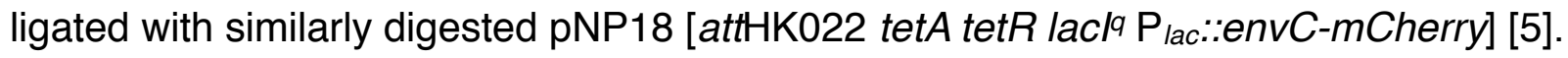

pMT94

The plasmid pMT94 [cat lacl Plac::nativeRBS_ybgC_tolQRA] was constructed as follows and involved several steps to assemble. First, the lac/q/Plac containing $\mathrm{Bg} / \mathrm{ll} / \mathrm{Xbal}$

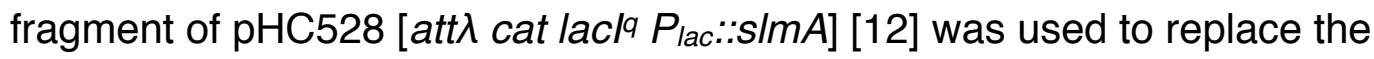
corresponding fragment of pDY31 [cat $\mathrm{P}_{\text {ara }}:$ nativeRBS-amiB] [13]. The primers 5'-

GTCAICTAGACTCTAACTTTTTGTTGCATTACCGGGATG-3' and 5'GTCA ATCGATTTACGGTTTGAAGTCCAATGGCG-3' were used to amplify nativeRBS_ybgC_tolQRA from genomic DNA. The resulting PCR product was purified, digested with Xbal and Clal and ligated into the above plasmid digested with the same enzymes to create pMT94. 
pMT101

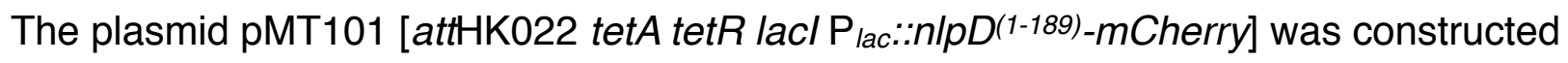
as follows. The primers 5'-TTAGGCACCCCAGGCTTTACAC-3' and 5'-

GTCACTCGAGAACTCCTTGCTCTGCTGCGTC-3' were used to amplify nlpD(1-189)

from pMT20. The resulting PCR product was purified, digested with Xbal and Xhol and ligated with similarly digested pNP18.

pMT102

The plasmid pMT102 [attHK022 tetA tetR lacl $\mathrm{P}_{\text {lac::nlpD(1-189)] was constructed as }}$ follows. The primers 5'-TTAGGCACCCCAGGCTTTACAC-3' and 5'GTCA from pMT20. The resulting PCR product was purified, digested with Xbal and HindIII and ligated with similarly digested pNP20.

pMT103

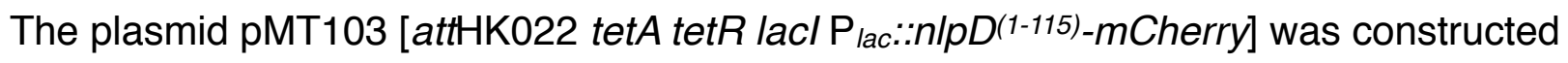
as follows. The primers 5'-TTAGGCACCCCAGGCTTTACAC-3' and 5'-

GTCA CTCGAGTTTCGGAATGTTCCCATACTGACGG-3' were used to amplify nlpD(1-115) from pMT20. The resulting PCR product was purified, digested with Xbal and Xhol and ligated with similarly digested pNP18.

pMT104 
The plasmid pMT104 [attHK022 tetA tetR lacl Plac::n/pD(1-115)] was constructed as follows. The primers 5'-TTAGGCACCCCAGGCTTTACAC-3' and 5'GTCAAAGCTTATTTCGGAATGTTCCCATACTGACGG-3' were used to amplify $n I p D(1-115)$ from $\mathrm{pMT} 20$. The resulting PCR product was purified, digested with Xbal and HindIII and ligated with similarly digested pNP20.

pMT105

The plasmid pMT105 [attHK022 tetA tetR lacl $\mathrm{P}_{\text {lac:: }}{ }^{s s} d s b A-n / p D^{(102-175)}$ ] was constructed as follows. The primers 5'-

GTCAGGATCCGGACGCATCGTCTATAACCGTCAGTATG-3' and 5'-

GTCAAAGCTTCCGCCGATTTATCGCTGC-3' were used to amplify nIpD(102-175) from genomic DNA. The resulting PCR product was purified, digested with BamHI and HindIII and ligated with similarly digested pNP19 [attHK022 tetA tetR lacl $\mathrm{P}_{l a c}:: s s d s b A-a m i B-$ sfGFP] [5].

pMT121

The plasmid pMT121 [attHK022 tetA tetR lacl $\left.\mathrm{P}_{\text {lac::: }}{ }^{s} d s b A-n / p D(27-379)\right]$ was constructed as follows. The $n / p D$ containing Xbal/Hindlll fragment of pMT18 [attHK022 bla lacla $\mathrm{P}_{\text {lac:: }}$ $\left.{ }^{s s} d s b A-n / p D^{(27-379)}\right]$ was used to replace the corresponding fragment of pNP20.

\section{pMT147}

The plasmid pMT147 [attHK022 tetA tetR lacl $\left.\mathrm{P}_{l a c}:: s s d s b A-n l p D^{(27-379)}-m C h e r r y\right]$ was constructed as follows. The primers 5'-TTAGGCACCCCAGGCTTTACAC-3' and 5'- 
GTCACTCGAGTCGCTGCGGCAAATAACGC-3' were used to amplify ${ }^{s s} d s b A$ nIpD(27-379) from pMT121. The resulting PCR product was purified, digested with Xbal and Xhol and ligated with similarly digested pNP20.

pMT149

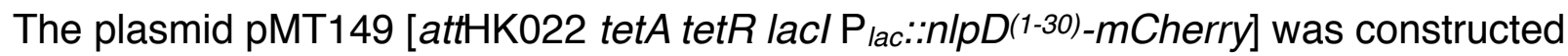
as follows. The primers 5'-TTAGGCACCCCAGGCTTTACAC-3' and 5'-

GTCACTCGAGTGAAGTGTCAGAACAGCCTGCC-3' were used to amplify n/pD(1-30) from pMT20. The resulting PCR product was purified, digested with Xbal and Xhol and ligated with similarly digested pNP20.

pMT178

The plasmid pMT178 [attHK022 tetA tetR lacl $\mathrm{P}_{l a c::}$ ss $\left.d s b A-n l p D(102-175)-m C h e r r y\right]$ was constructed as follows. The primers 5'-TTAGGCACCCCAGGCTTTACAC-3' and 5'GCTACTCGAGGCCAGTGATTGGCGTACCG-3' were used to amplify ssdsbA$n / p D(102-175)$ from $p M T 105$. The resulting PCR product was purified, digested with Xbal and Xhol and ligated with similarly digested pNP20.

pMT179

The plasmid pMT179 [attHK022 tetA tetR lacl $\mathrm{P}_{\text {lac:: }}{ }^{s s} d s b A-n / p D^{(250-379)}$ ] was constructed as follows. The primers 5'-GTCAGGATCCAGTACATCAACCAGTACGCCTATCTCC-3' and 5'-GACGAAAGTGATTGCGCCTACC-3' were used to amplify $n / p D(250-379)$ from 
pMT20. The resulting PCR product was purified, digested with BamHI and HindIII and ligated with similarly digested pNP19.

pMT180

The plasmid pMT180 [attHK022 tetA tetR lacl $\left.\mathrm{P}_{\text {lac:: }}{ }^{s s} d s b A-n / p D^{(250-379)}-m C h e r r y\right]$ was constructed as follows. The primers 5'-TTAGGCACCCCAGGCTTTACAC-3' and 5'GTCACTCGAGTCGCTGCGGCAAATAACGC-3' were used to amplify $n / p D(250-379)$ from pMT179. The resulting PCR product was purified, digested with Xbal and Xhol and ligated with similarly digested pNP20.

pMT181

The plasmid pMT181 [attHK022 tetA tetR lacl $\mathrm{P}_{\text {lac:: }}{ }^{s s} d s b A-n / p D^{(189-379)]}$ was constructed as follows. The primers 5'-GTCAGGATCCGTTGTGATCAAGCCTGCACAAAATTCC-3' and 5'-GACGAAAGTGATTGCGCCTACC-3' were used to amplify nlpD(189-379) from pMT20. The resulting PCR product was purified, digested with BamHI and HindIII and ligated with similarly digested pNP19.

\section{pMT182}

The plasmid pMT182 [attHK022 tetA tetR lacl $\mathrm{P}_{\text {lac:: }}{ }^{s s} d s b A-n l p D^{(189-379)-m C h e r r y] ~ w a s ~}$ constructed as follows. The primers 5'-TTAGGCACCCCAGGCTTTACAC-3' and 5'GTCACTCGAGAACTCCTTGCTCTGCTGCGTC-3' were used to amplify n/pD(189-379) from pMT181. The resulting PCR product was purified, digested with Xbal and Xhol and ligated with similarly digested pNP20. 
pMT187

This plasmid pMT187 [aadA repA(ts) lacla $\mathrm{P}_{\text {lac }:: e n v C-L E}$ Scel cl857 $\left.\mathrm{P}_{\lambda \mathrm{R}}:: \mathrm{i}-\mathrm{scel}\right]$ is a derivative of pBL200 [aadA repA(ts) $\mathrm{P}_{\text {syn135::ftsN Scel }}$ cl857 $\mathrm{P}_{\lambda \mathrm{R}}:$ :i-scel] [14] in which the

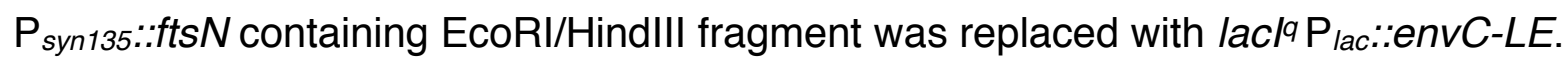

pMT196

The plasmid pMT196 [atth cat lacl Plac::yraP(1-191)] was constructed as follows. The primers 5'-

GCTA TCTAGATTAAGAAGGAGATATACATATGAAGGCATTATCGCCAATCGC-3' and 5'-GCTA yraP(1-191) from genomic DNA. The resulting PCR product was purified, digested with

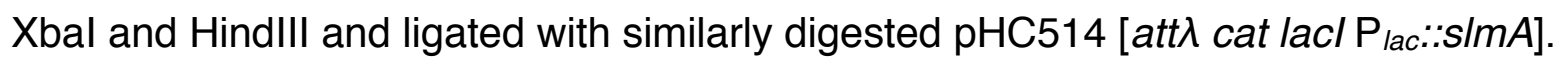

pMT197

The plasmid pMT197 [atth cat lacl Plac::yraP(1-191)-mCherry] was constructed as follows. The primers 5'-

GCTA TCTAGATTAAGAAGGAGATATACATATGAAGGCATTATCGCCAATCGC-3' and 5'-GCTA CTCGAGTTTAATAAACGTAAACGCCGTAGTTACCCG-3' were used to amplify yraP(1-191) from genomic DNA. The resulting PCR product was purified, digested with Xbal and Xhol and ligated with a similarly digested pHC514 derivative containing mCherry. 
pMT198

The plasmid pMT198 [att入 cat lacl $\mathrm{P}_{\text {lac:: }}$ yraP(VA-20,21-DE)] was constructed as follows. The primers 5'-

GCTAICTAGATTAAGAAGGAGATATACATATGAAGGCATTATCGCCAATCGC-3'/5'CTACGGCAGCCTCATCACAACCTTGCAACAGCAGCG-3' and 5'GCAAGGTTGTGATGAGGCTGCCGTAGTGGGTACCGC-3'/5'GCTAAAGCTTGGCTGCTATTTAATAAACGTAAACGCCG-3' were used in overlap extension PCR to amplify the yraP gene from genomic DNA and to perform site directed mutagenesis to create the (VA-20,21-DE) mutations in the yraP gene. The resulting PCR product was purified, digested with Xbal and HindIII and ligated with similarly digested pHC514 [atth cat lacl Plac::s/mA].

pMT199

The plasmid pMT199 [atth cat lacl $\mathrm{P}_{\text {lac:: }}$ yraP(VA-20,21-DE)-mCherry] was constructed as follows. The primers 5'-

GCTAICTAGATTAAGAAGGAGATATACATATGAAGGCATTATCGCCAATCGC-3'/5'CTACGGCAGCCTCATCACAACCTTGCAACAGCAGCG-3' and 5'GCAAGGTTGTGATGAGGCTGCCGTAGTGGGTACCGC-3'/5'GCTACTCGAGTTTAATAAACGTAAACGCCGTAGTTACCCG-3' were used in overlap extension PCR to amplify the yraP gene from genomic DNA and to perform site directed mutagenesis to create the (VA-20,21-DE) mutations in the yraP gene. The resulting PCR product was purified, digested with Xbal and Xhol and ligated with a similarly digested pHC514 derivative containing mCherry. 
pMT209

The plasmid pMT209 [att入 cat lac/ $\mathrm{P}_{\text {lac::: }}{ }^{s} d s b A-y r a P^{(24-191)}$ ] was constructed in two steps as follows. The primers 5'-GCTAGGATCCGTAGTGGGTACCGCTGCTG-3' and 5'GCTAAAGCTTGGCTGCTATTTAATAAACGTAAACGCCG-3' were used to amplify yraP(24-191) from genomic DNA. The resulting PCR product was purified, digested with BamHI and HindIII and ligated with similarly digested pNP19 to make pMT200 [attHK022 tetA tetR lac/ $\mathrm{P}_{\text {lac:: }}{ }^{\text {ss } d s b A-y r a P(24-191)]}$. The $y r a P$ containing $\mathrm{Xbal} / \mathrm{Hindlll}$ fragment of pMT200 was then used to replace the corresponding fragment of pHC514.

pMT210

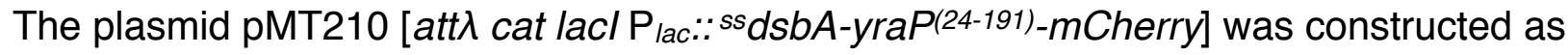
follows. The primers 5'-TTAGGCACCCCAGGCTTTACAC-3' and 5'GCTACTCGAGTTTAATAAACGTAAACGCCGTAGTTACCCG-3' were used to amplify ${ }^{s s} d s b A-y r a P(24-191)$ from pMT200. The resulting PCR product was purified, digested with Xbal and Xhol and ligated with a similarly digested pHC514 derivative containing mCherry.

\section{pMT224}

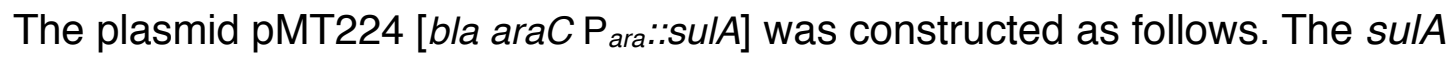

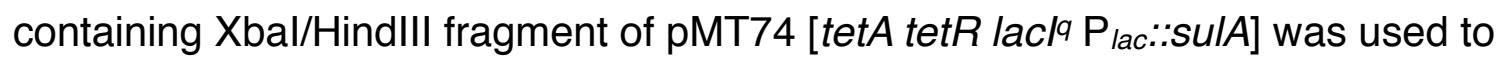
replace the corresponding fragment of pBAD24 [bla araC $P_{\text {ara:::empty] [6]. }}$ 


\section{REFERENCES}

1. Guyer MS, Reed RR, Steitz JA, Low KB. Identification of a sex-factor-affinity site in E. coli as gamma delta. Cold Spring Harb Symp Quant Biol. 1981;45 Pt 1:13540.

2. Johnson JE, Lackner LL, Hale CA, de Boer PAJ. ZipA is required for targeting of DMinC/DicB, but not DMinC/MinD, complexes to septal ring assemblies in Escherichia coli. J Bacteriol. 2004 Apr 1;186(8):2418-29.

3. Bernhardt TG, de Boer PAJ. Screening for synthetic lethal mutants in Escherichia coli and identification of EnvC (YibP) as a periplasmic septal ring factor with murein hydrolase activity. Mol Microbiol. 2004 Jun;52(5):1255-69.

4. Uehara T, Dinh T, Bernhardt TG. LytM-domain factors are required for daughter cell separation and rapid ampicillin-induced lysis in Escherichia coli. J Bacteriol. 2009 Aug;191(16):5094-107.

5. Peters NT, Dinh T, Bernhardt TG. A fail-safe mechanism in the septal ring assembly pathway generated by the sequential recruitment of cell separation amidases and their activators. J Bacteriol. 2011 Sep;193(18):4973-83.

6. Guzman LM, Belin D, Carson MJ, Beckwith J. Tight regulation, modulation, and high-level expression by vectors containing the arabinose PBAD promoter. $\mathrm{J}$ Bacteriol. 1995 Jul 1;177(14):4121-30. 
7. Haldimann A, Wanner BL. Conditional-replication, integration, excision, and retrieval plasmid-host systems for gene structure-function studies of bacteria. $\mathrm{J}$ Bacteriol. 2001 Nov 1;183(21):6384-93.

8. Datsenko KA, Wanner BL. One-step inactivation of chromosomal genes in Escherichia coli K-12 using PCR products. Proc Natl Acad Sci USA. 2000 Jun 6;97(12):6640-5.

9. Tsang M-J, Bernhardt TG. A role for the FtsQLB complex in cytokinetic ring activation revealed by an ftsL allele that accelerates division. Mol Microbiol. 2015 $\operatorname{Mar} ; 95(6): 925-44$.

10. Bernhardt TG, de Boer PAJ. SImA, a nucleoid-associated, FtsZ binding protein required for blocking septal ring assembly over Chromosomes in E. coli. Mol Cell. 2005 May 27;18(5):555-64.

11. Uehara T, Parzych KR, Dinh T, Bernhardt TG. Daughter cell separation is controlled by cytokinetic ring-activated cell wall hydrolysis. EMBO J. 2010 Apr $21 ; 29(8): 1412-22$.

12. Cho H, McManus HR, Dove SL, Bernhardt TG. Nucleoid occlusion factor SImA is a DNA-activated FtsZ polymerization antagonist. Proc Natl Acad Sci USA. 2011 Mar 1;108(9):3773-8.

13. Yang DC, Tan K, Joachimiak A, Bernhardt TG. A conformational switch controls cell wall-remodelling enzymes required for bacterial cell division. Mol Microbiol. 2012 Aug;85(4):768-81. 
14. Liu B, Persons L, Lee L, de Boer PAJ. Roles for both FtsA and the FtsBLQ subcomplex in FtsN-stimulated cell constriction in Escherichia coli. Mol Microbiol. 2015 Mar;95(6):945-70. 